Meta

Journal des traducteurs

Translators' Journal

\title{
Le rapporteur et l'interprète de conférence
}

\section{Térence Macnamee}

Volume 44, numéro 2, juin 1999

URI : https://id.erudit.org/iderudit/003533ar

DOI : https://doi.org/10.7202/003533ar

Aller au sommaire du numéro

\section{Éditeur(s)}

Les Presses de l'Université de Montréal

ISSN

0026-0452 (imprimé)

1492-1421 (numérique)

Découvrir la revue

Citer cet article

Macnamee, T. (1999). Le rapporteur et l'interprète de conférence. Meta, 44(2), 280-294. https://doi.org/10.7202/003533ar

\section{Résumé de l'article}

On a caractérisé le traducteur-interprète comme un rapporteur. Cet article étudie le travail du rapporteur de conférence à la lumière de la réflexion sur le travail de l'interprète de conférence. Je décris une méthode de travail pour le rapporteur qui participe à des activités de consultation publique ou interne. Le rapporteur travaille en deux temps: en temps réel, non réversible, dans la réunion, et en temps réversible, après la réunion, lorsqu'il procède à l'analyse des données. Cette analyse se fait par des techniques linguistiques. Le travail en deux temps est rendu possible par l'ordinateur. La formation des rapporteurs à l'avenir devrait ressembler à celle que l'on fournit aux futurs interprètes de conférence.
Ce document est protégé par la loi sur le droit d'auteur. L'utilisation des services d'Érudit (y compris la reproduction) est assujettie à sa politique d'utilisation que vous pouvez consulter en ligne.

https://apropos.erudit.org/fr/usagers/politique-dutilisation/ 


\title{
Le rapporteur et l'interprète de conférence
}

\author{
tér ence macnamee
}

Dublin, Irlande

\begin{abstract}
RÉSUMÉ
On a caractérisé le traducteur-interprète comme un rapporteur. Cet article étudie le travail du rapporteur de conférence à la lumière de la réflexion sur le travail de l'interprète de conférence. Je décris une méthode de travail pour le rapporteur qui participe à des activités de consultation publique ou interne. Le rapporteur travaille en deux temps: en temps réel, non réversible, dans la réunion, et en temps réversible, après la réunion, lorsqu'il procède à l'analyse des données. Cette analyse se fait par des techniques linguistiques. Le travail en deux temps est rendu possible par l'ordinateur. La formation des rapporteurs à l'avenir devrait ressembler à celle que l'on fournit aux futurs interprètes de conférence.
\end{abstract}

\begin{abstract}
The translator-interpreter has been characterised as a rapporteur. This article studies the work of the conference rapporteur in the light of what has been written about the work of the conference interpreter. I outline a working method for the rapporteur who participates in public or internal consultation activities. The rapporteur operates in two sorts of time: in real (non-reversible) time, during the actual meeting, and in reversible time, after the meeting, when he does his analysis of the data. This analysis is done by linguistic techniques. The working in two sorts of time is made possible by the computer. The training of rapporteurs in the future should emulate that provided to intending conference interpreters.
\end{abstract}

Dans des discussions assez récentes, on a eu tendance à comparer le travail du traducteur, de l'interprète, à la réexpression du contenu intralingual. On a parlé du traducteur, de l'interprète comme rapporteur.

Seleskovitch (1968), Lederer (1981) et Seleskovitch et Lederer (1986) ont écrit que l'interprète de conférence rend le sens global de l'acte de communication et non la signification des mots. L'âme de son travail consiste à comprendre, et à faire comprendre. C'est-à-dire que l'interprète se trouve en quelque sorte dans la situation de quelqu'un qui rapporte ce qui a été dit, qui donne un compte rendu du contenu. Seleskovitch et Lederer (1986: 18) disent en effet:

$\mathrm{N}$ 'oublions pas que le besoin de la traduction découle directement du besoin de communication et que celui-ci existe tout autant à l'intérieur d'une même langue où la communication se passe d'intermédiaire, qu'entre deux langues où la médiation du traducteur devient nécessaire. N'est-il pas al ors légitime de penser que le processus de la communication tel qu'il s'effectue à l'intérieur d'une seule et même langue est le même que celui qui relie le traducteur à son texte original, puis sa traduction au lecteur qui en prendra connaissance, de sorte que le processus de la traduction relève beaucoup plus d'opérations de compréhension et d'expression que de comparaisons entre les langues.

Mossop $(1984,1987)$ affirme que le traducteur ne se contente pas de transmettre mécaniquement, ni de traiter des mots: il évalue, sélectionne ce qu'il croit pertinent 
dans les énoncés du texte de départ. C'est un rapporteur, qui reformule ce qui a été dit, qui travaille à partir des formules du discours rapporté. II participe pleinement à la transaction communicative.

Folkart (1991) accepte cette notion du traducteur rapporteur comme point de départ de sa discussion critique de la traduction. II s'agit, selon elle, dans la traduction d'un discours rapporté, qui n'est pas «transparent» comme le voudrait un certain «mythe de la traduction». Folkart a «choisi de situer la traduction par rapport à cette autre manifestation du vouloir-redire qu'est le discours rapporté intralingual » (1991: 13). Elle parle du travail du traducteur et du rapporteur comme réénonciation d'un déjà-dit.

Une synthèse de ces approches serait de dire que l'interprète ou le traducteur n'est pas un simple transmetteur ou transcodeur qui s'efface en transmettant le message. C'est un rapporteur en effet, qui construit le sens de ce qui a été dit à partir de sa compréhension de celui-ci, qui ajoute dans une certaine mesure sa perspective au message, et qui est donc «présent» dans sa traduction.

Or, cette discussion m'a été très utile dans la mise au point d'une description du travail du rapporteur de conférence et je compte la citer au cours de la discussion qui suit sur le rôle du rapporteur. Je constate que, si le traducteur-interprète est un rapporteur, le rapporteur lui aussi est comme un interprète, spécifiquement un interprète de conférence.

Le mot «rapporteur » s'emploie aujourd'hui pour désigner l'activité de celui ou de celle qui note le contenu d'une réunion, d'une consultation, pour donner un compte rendu de la discussion une fois la séance terminée. On compte typiquement sur le rapporteur pour mettre une cohérence dans les discussions et en tirer les conclusions qui s'imposent. Le rôle du rapporteur s'avère particulièrement important dans les activités de consultation de groupe. Tenant compte de l'importance accrue de la consultation dans la vie publique ${ }^{1}$, je crois qu'il serait temps d'examiner le rôle du rapporteur et la nature de son travail.

Ce sont les gouvernements et les grandes entreprises publiques, en effet, qui sont les clients typiques du rapporteur, lorsqu'ils entreprennent des consultations. II y en a deux sortes: tout d'abord, la consultation externe, où l'organisation consulte les citoyens pour savoir leur opinion sur un sujet quelconque; ensuite, la consultation interne, où l'organisation consulte ses fonctionnaires pour avoir leur contribution aux grandes décisions.

Dans toute consultation, il faut:

- entendre ce que disent les participants;

- comprendre ce qu'ils veulent dire.

Souvent aussi, dans la consultation, il y a l'ambition d'avoir un effet sur ce qui s'exprime, pour que les participants comprennent les enjeux, pour que le dialogue s'engage, enfin pour qu'on aboutisse à un consensus sur le plan d'action à suivre.

Pour réussir ces ambitions, il faut une technique d'enregistrement et d'analyse des données de la consultation, de ce qui se dit dans les réunions. Toutefois, il n'existe pas de norme pour l'en registrement et l'analyse dans de telles circonstances. Certains groupes (comme des comités de parlementaires) ont recours à la sténographie, l'enregistrement mot à mot, comme les tribunaux. Plus souvent, c'est la personne qui dirige la réunion, ou une secrétaire, qui prend des notes au fur et à mesure. 
Aucune de ces deux solutions n'est satiffaisante à vrai dire. Le mot à mot, c'est trop. Prendre des notes en passant, c'est trop peu. Afin de pouvoir proposer une solution adéquate, j'ai voulu systématiser le rôle du rapporteur.

Dans ce qui suit, je décris l'activité du rapporteur telle que je la pratique depuis une dizaine d'années. La méthode que j'ai élaborée consiste à noter ce qui se dit pendant la réunion sur un ordinateur personnel; à intervenir de temps en temps dans la réunion même, en utilisant un rétroprojecteur, pour montrer aux participants ce qu'ils ont dit, dans le texte de mes notes; et à leur fournir un rapport intérimaire imprimé, le lendemain de la réunion, et un rapport final à la fin de la série de réunions s'il y en a. J'ai pu développer cette méthode en ayant recours au modèle de l'interprète de conférence.

\section{LE TRAVAIL D'ENREGISTREMENT DU RAPPORTEUR EN TEMPS RÉEL}

\section{La quête du sens}

Comme l'interprète de conférence, le rapporteur opère en temps réel, dans une situation de réunion. II écoute l'énoncé de l'orateur, et le rend aux participants dans une autre forme: dans le cas de l'interprète, c'est dans une langue seconde; dans le cas du rapporteur, c'est sous la forme de l'écrit. La traduction, mais le rapport aussi, est une «reprise faisant intervenir un changement de médium linguistique» (Folkart 1991 : 77).

Dans l'un et l'autre cas, c'est le sens de l'énoncé qui est transféré, non pas les mots. Seleskovitch (1976) évoque le proverbe verba volant, scripta manent, et le modifie dans le cas de l'interprète à verba volant, sensus manet. Seleskovitch et Lederer définissent le sens comme «la rencontre dans l'esprit de la formulation linguistique [de l'orateur] et des connaissances dont on dispose [à l'écoute] » (1986: 22), et elles précisent: «Élément central des rapports entre les hommes, le sens [...] est également l'objet de la traduction » (1986: 18). La traduction est une réexpression du sens. Le rapport aussi.

Ce sens consiste en discussions, en arguments. Seleskovitch et Lederer définissent «l'unité de sens» comme «le segment de discours dont l'avancée à un moment donné fait prendre conscience à l'auditeur ou au lecteur du vouloir-dire désigné par la formulation linguistique» (1986: 268).

Rappelons que l'ethnologue qui enregistre les mythes des informateurs indigènes et qui les analyse par la suite se concentre lui aussi sur le sens, selon Lévi-Strauss (1958: 232) qui précise:

On pourrait définir le mythe comme ce mode de discours où la valeur de la formule traduttore, traditore tend à zéro [...] La val eur du mythe comme mythe persiste, en dépit de la pire traduction [...] La substance du mythe ne se trouve pas dans le style, ni dans le mode de narration, ni dans la syntaxe, mais dans l'histoire qui y est racontée. Le mythe est langage; mais un langage qui travaille à un niveau très élevé, et où le sens parvient, si l'on peut dire, à décoller du fondement linguistique sur lequel il a commencé par rouler.

Ce niveau très élevé, c'est évidemment le niveau de représentation du sens. LéviStrauss, dans le même texte, identifie l'idéologie politique, lieu privilégié des croyances 
et des valeurs, comme l'équivalent du discours mythique dans notre société contemporaine. Le rapporteur, nous le verrons, a pour tâche de dégager ce sens «idéologique», ces croyances et valeurs, explicites ou implicites, qui déterminent le vouloir-dire des participants dans une discussion.

Dans la perspective de Seleskovitch, la parole de l'orateur laisse une trace chez l'interprète ou le rapporteur, qui est la compréhension. Le rapporteur, on peut même dire, comme le traducteur selon Folkart (1991) d'ailleurs, est celui qui s'approprie le message, et qui le «réénonce» ou le retransmet avec la «valeur ajoutée» de sa compréhension interprétative. Le message d'arrivée contient donc la trace de l'activité du rapporteur. S'élevant contre «le topos de l'effacement du traducteur » (1991: 12), Folkart déclare: «On ne saurait de ce fait réénoncer sansy mettre du sien » (1991: 14). Le rapporteur ou le traducteur, quand ils réénoncent ce qui a été dit, se réapproprient l'énoncé de l'orateur original. «Réappropriation » est définie de la manière suivante:

le processus moyennant lequel le réénonciateur fait sien l'énoncé qu'il restituera sous forme de discours rapporté ou de traduction, processus qui intervient dans toute réception et qui implique un filtrage inéluctable à travers le sujet récepteur [...] La réénonciation constitue toujours une réappropriation. (1991: 450)

Le rapporteur ne se contente pas de retransmettre le message de l'émetteur original ; il le forme et le re-forme, il y laisse la trace de sa présence. Dans un certain sens, il se l'approprie, même, à ses propres fins qui ne sont pas celles de l'intervenant individuel. L'émetteur original ne pense qu'à ses propos et à la manière de les exprimer aux autres, sinon de persuader ceux-ci de la justesse de ses opinions, alors que le rapporteur tente d'harmoniser ce qui vient d'être dit avec ce qui a déjà été dit, et de replacer cet énoncé dans le champ des significations déjà énoncées. Le rapporteur est là justement comme professionnel du langage pour redire le déjà-dit et le dire mieux peut-être qu'il n'a été dit au départ, et pour harmoniser les énoncés du déjà-dit en un discours unifié.

C'est dans ce sens que le rapporteur peut «améliorer » les discussions des sujets parlants. Jusqu'à quel point? L'essentiel, c'est d'interpréter sans trahir. Mais voici le point, sans doute, où le rôle du rapporteur se distingue de celui de l'interprète, du traducteur consciencieux. Seleskovitch et Lederer (1986) affirment: "Le sens d'une phrase est ce qu'un auteur veut délibérément exprimer, ce n'est pas la raison pour laquelle il parle, les causes ou les conséquences de ce qu'il dit. Le sens ne se confond pas avec des mobiles ou des intentions. Le traducteur qui se ferait exégète, l'interprète qui se ferait herméneute transgresserait les limites de ses fonctions. » $M$ ais le rapporteur, lui, se fait herméneute. Sinon dans un premier temps, celui de la réunion, au moins dans un deuxième, celui de l'analyse. Et même dans la réunion, le rapporteur va souvent plus loin que le sens tel que défini par Seleskovitch. II redit les choses que les participants disent «mieux qu'ils ne pourraient le dire eux-mêmes», et il confronte les participants à l'enregistrement de leurs propos. C'est intervenir plus activement, plus radicalement dans le processus de la réunion que ne ferait certes l'interprète de conférence.

D'un autre côté, le rapporteur fera bien de retenir les mots et les expressions, les poncifs utilisés par les participants, pour mieux comprendre leur pensée intime. II peut faire cela assez facilement, puisqu'il ne traduit pas mais prend des notes dans la même langue. 
Selon M ossop (communication personnelle), le trait distinctif de la traduction, c'est l'attention faite aux mots. Le traducteur, l'interprète, construit sa traduction non seulement à partir du sens, mais aussi à partir d'un certain nombre de mots apparaissant dans l'énoncé de départ, la mémoire à court terme assurant la conservation de ceux-ci surtout dans le cas del'interprétation. C'est là une formulation que le rapporteur lui aussi peut retenir.

Pourquoi le rapporteur, dans son travail de réappropriation et de réénonciation, respecterait-il les mots utilisés par les participants? Parce qu'il veut soumettre le texte noté, on le verra, à une analyse ultérieure, afin de dégager des couches plus profondes de sens. Pour ce faire, il s'occupe dès le départ des mots, de la terminologie qu'utilisent les participants, puisque la terminologie fournit la clef des notions et des lieux communs, même des croyances et des valeurs, qui déterminent le discours (comme j'ai voulu montrer dans MacNamee 1984).

\section{Technique de la prise de notes}

Au fur et à mesure que les participants discutent, le rapporteur enregistre le sens de la discussion dans son ordinateur. II prend des notes. Quelle est la nature de ces notes?

Le rapporteur note, en premier lieu, les opinions formulées par les participants, ce que j'appelle les énoncés. M ais comment choisir ces énoncés, à partir du flot de la discussion? II faut opérer une sélection, bien sûr, puisqu'on n'enregistre pas mot à mot. Les énoncés sont les «unités de sens», et rappelons que Seleskovitch et Lederer définissent «l'unité de sens» comme «le segment de discours dont l'avancée à un moment donné fait prendre conscience à l'auditeur ou au lecteur du vouloir-dire désigné par la formulation linguistique» (1986: 268). II faut choisir des énoncés qui font avancer la discussion: propositions nouvelles, arguments qui soutiennent ou contre-arguments qui s'opposent à une proposition antérieure; ou alors, position d'un problème, ou mention d'une notion nouvelle. Des formules, bref, qui ont des rapports logiques entre elles. Les rapports logiques que l'on identifie entre les énoncés de différents orateurs aident à préparer le consensus, par degrés.

Comme nous avons déjà dit, on trouvera, en écoutant, que les sujets parlants dans leurs énoncés utilisent des mots qui sont ou des termes techniques propres au discours en question, ou alors des termes qui ne sont pas techniques mais qui possèdent manifestement une signification particulière pour ces sujets, parce qu'ils identifient une notion importante dans ce discours. Ces deux sortes de termes ou mots-clés, on pourra les reconnaître lorsqu'un orateur les évoque à plusieurs reprises, ou que plusieurs orateurs les évoquent séparément, ou qu'ils apparaissent dans des contextes où ils ne peuvent pas avoir seulement la signification quotidienne ou usuelle. Ces mots-clés sont à noter soigneusement par le rapporteur, puisqu'ils révèlent la présence de ce que j'appelle les notions du discours.

Écartons une méprise que l'on entend souvent à propos de ce que fait le rapporteur lorsqu'il prend ses notes. II ne s'agit pas d'un procès-verbal de la réunion. Un procès-verbal est:

1. un compte rendu des décisions, alors que le texte du rapporteur rapporte toute la discussion; 
2. une activité passive d'enregistrement, alors que le rapporteur intervient activement avec son feed-back dans la réunion même;

3. un texte final, clos, sans suite (sauf modifications éventuelles), alors que le texte du rapporteur est un texte provisoire, ouvert, qui subira des transformations presque à l'infini.

C'est la comparaison avec l'interprète qui explique le fait que le rapporteur est beau coup plus qu'une secrétaire ou un sténographe. Le procès-verbal, c'est la «transparence» voulue, au sens de Folkart; le rapport, c'est une sélection, une activité, une opération. L'activité du rapport simultané n'est pas un enregistrement passif de ce qui se dit dans l'espace d'une rencontre; c'est une activité structurante, puisque le rapporteur forme et transforme les données de la discussion pour les analyser à son gré par la suite.

\section{Maïeutique}

Le rapporteur écoute ce que disent les participants. Sa seule présence, même silencieuse, change la situation. Comment cela? L'orateur est encouragé par le fait que quelqu'un écoute soigneusement, alors que les autres participants ne veulent probablement que faire valoir leur point de vue. Le rapporteur est cette présence silencieuse qui écoute et qui inspire les participants à exprimer leur pensée et à s'écouter les uns les autres. L'élément transformateur, c'est que quelqu'un est là qui écoute, totalement, et qui ne fait qu'écouter.

Celui qui écoute est un «maïeuticien », une sage-femme au sens consacré il y a belle lurette par Socrate: il aide le sujet parlant à comprendre ce que celui-ci a dans la tête et s'efforce d'exprimer. (Socrate, dans le Théétète de Platon, rappelle qu'il est le fils d'une sage-femme de renom, et qu'il exerce le même métier à sa façon, en aidant ses interlocuteurs à «enfanter » leurs notions.) II fait cela souvent en posant des questions. Mais, comme le note G. Corradi Fiumara (1990), il le fait surtout en montrant qu'il suit et comprend. Je trouve que le rapporteur face aux participants dans une réunion ne fait rien d'autre.

\section{Structure musicale}

Le rapporteur dans une réunion enregistre non un texte unique issu d'un seul auteur, non un ensemble de textes plus ou moins distincts sur le même sujet, mais un «texte» de groupe où l'on peut entendre les voix d'un certain nombre de personnes.

Nous l'avons vu tout à l'heure, le rapporteur cherche dans le texte du groupe des énoncés qui se complètent, se développent ou se contredisent, ou qui se succèdent après un hiatus. Ce «texte» du groupe offre donc des analogies avec une composition musicale. Je dis musicale parce que, au fond, les voix s'accordent et font un tout plus ou moins cohérent; ce ne sont pas des textes simultanés en concurrence, comme les émissions télévisées diffusées par des chaînes rivales.

On sait que Lévi-Strauss (1958: 234 sq.) a comparél'analyse d'un texte mythique à la lecture d'une partition. On doit selon lui pouvoir lire tel ou tel mythe non seulement horizontalement, un énoncé après l'autre, mais verticalement, en notant les similitudes, les harmonies justement entre deux ou plusieurs énoncés. L'on constate, dans une partition, «que certains groupes de notes se répètent à intervalles, de 
façon identique et partielle, et que certains contours mélodiques, apparemment éloignés les uns des autres, offrent entre eux des analogies... ». C'est en effet «le principe de ce que nous appelons harmonie: une partition d'orchestre n'a de sens que lue diachroniquement selon un axe (page après page, de gauche à droite), mais en même temps synchroniquement selon l'autre axe, de haut en bas».

Les participants dans une réunion concourent à l'élaboration du texte collectif en contribuant par des phrases, des propositions, en évoquant des préoccupations. Le rapporteur les note, les combine pour en faire un texte unique. Texte qui comprendra des contradictions, des controverses, des désaccords, des contrastes, comme les thèmes d'une composition musicale. Le rapporteur au clavier de son ordinateur est comme un soliste dans un concerto pour piano et orchestre - l'orchestre annonce le thème, le soliste le répète, le commente, le développe en dialogue avec l'orchestre. $C$ 'est ce que nous allons voir maintenant.

\section{Rétroaction}

Au fur et à mesure que les participants discutent, le rapporteur fait le sommaire des points soulignés ou des opinions exprimées. À un certain moment, dans une pause de la discussion, il projette ce texte par le moyen du rétroprojecteur sur le grand écran. Tous les participants le lisent. Ils voient tout de suite où ils en sont, et la direction que la discussion doit prendre par la suite. Le panneau de rétroprojection est donc l'outil indispensable du feed-back consécutif. C'est ainsi que le texte du rapporteur est le miroir du discours, ou un «verre grossissant» (dans la formule de Seleskovitch).

Cette lecture en commun s'accompagne typiquement des commentaires parlés du rapporteur. II pourra demander aux participants de préciser certaines formules, ou alors il pourra rendre explicites certains présupposés de la discussion, certaines valeurs qui semblent communes aux participants pour n'être pas évoquées. Autre intervention capitale: il peut faire ressortir une opinion qui a été exprimée par un participant mais négligée par les autres, parce qu'il y a malheureusement dans la dynamique des groupes des mécanismes d'exclusion, contre lesquels il faut lutter.

L'activité de rétroaction se fait en temps réel. Pourquoi ? Pour donner du feedback aux participants tout de suite, et donc pour avoir un effet sur les progrès ultérieurs de la discussion. On peut envisager le processus de rétroaction comme une sorte de convergence des fils discursifs, comme ceci :

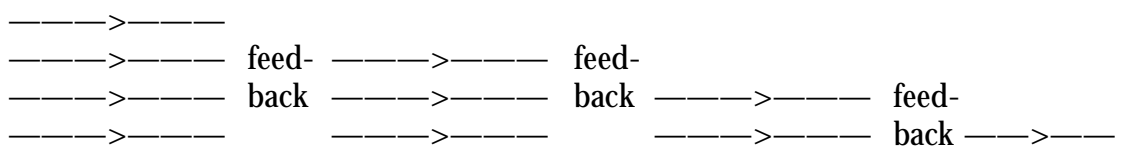

\section{Texte, élément fusible}

Depuis H omère on parle de «épea pteróenta » ou paroles ailées. Le proverbe cité par Seleskovitch dit: Verba volant, scripta manent. La parole est un élément volatil, puisqu'elle se perd presque immédiatement une fois dite; mais une fois captée sur l'écran de l'ordinateur, c'est un élément fusible, puisqu'elle est saisie au passage, pour ainsi dire, et formée en discours, entre les mains du rapporteur. 
Le texte de la discussion est comme de l'or ou du verre en fusion. Le rapporteur est comme l'ouvrier qui le reçoit dans cette condition et, pendant qu'il se laisse plier et former, en temps réel donc, il l'exprime dans des formules concises et précises qui en captent le sens.

Voilà en effet le travail accompli par le rapporteur en temps réel. II laisse sa trace, une valeur ajoutée, dans le message qu'il réénonce, selon la perspective de Folkart qui précise: «on ne saurait assimiler ni la réénonciation en général ni la traduction en particulier à un non-travail » (1991: 358). Le message rapporté est tranforformé une fois pour toutes, parce que «Qui dit travail dit irréversibilité. » (1991: 359)

\section{Les deux temps}

Dans une discussion restée classique, Lévi-Strauss (1958) a écrit qu'il y a deux temps dans le langage, ce qu'il appelle le temps réversible et le temps irréversible. Cela correspond à la dichotomie saussurienne de la langue et de la parole. «La langue appartient au domaine d'un temps réversible, et la parole, à celui d'un temps irréversible» (1958: 230). La langue, objet d'étude, structure virtuelle, existe dans le temps réversible; mais la parole, qui se dit et s'évanouit aussitôt et ne peut pas être rappelée, existe dans le temps irréversible. (Verba volant.) M ais Lévi-Strauss va plus loin, affirmant que le texte (et c'est bien du texte mythique qu'il s'agit dans la discussion de l'ethnologue), s'il appartient au domaine de la parole et s'il existe en temps irréversible, appartient aussi au domaine du temps réversible, puisqu'il se prête à l'analyse thématique, rhétorique, etc. : «Or, le mythe se définit aussi par un système temporel, qui combine les propriétés des deux autres. Un mythe se rapporte toujours à des évènements passés [...] M ais la valeur intrinsèque attribuée au mythe provient de ce que ces évènements, censés se dérouler à un moment du temps, forment aussi une structure permanente» (1958: 231). (Sensus manet.) II précise en outre: «Ce système est en effet à deux dimensions: à la fois diachronique et synchronique, et réunissant ainsi les propriétés caractéristiques de la “langue" et de la "parole” » (1958: 234).

Le temps irréversible est ce que les informaticiens (et les interprètes de confé rence) appellent le temps réel. Le temps réversible est un temps virtuel, où les structures permanentes du déjà-dit peuvent se révéler à la réflexion. Les discussions, les conversations, dans la mesure où ce sont des textes, existent dans ces deux temps. Le rapporteur s'en rend compte très clairement. II prend ses notes en temps réel, irréversible; mais ces notes existeront désormais en temps réversible, dans la mémoire de l'ordinateur, et pourront subir toutes sortes de transformations.

\section{Rôle de l'ordinateur}

L'ordinateur permet l'exploitation des deux temps du langage que nous avons évoqués. Grâce à l'enregistrement, le texte de la discussion, qui existait dans le temps irréversible, se double d'une existence en temps réversible. On peut dire cela, il est vrai, de tout texte écrit. Mais le texte capté sur ordinateur est vraiment un élément fusible. Une fois un texte emmagasiné dans la mémoire de l'ordinateur, on pourra le modifier, l'étendre, le raccourcir, l'adapter de diverses façons, le transformer presque à l'infini. Le texte informatisé n'est jamais une entité close, finie. L'enregistrement du 
sens des énoncés en temps réel, irréversible, permet son analyse par la suite, son développement, sa transformation, en temps réversible.

Nous voyons aujourd'hui la parole, l'écrit éphémère, se doubler d'une transcription, d'un enregistrement ou d'une analyse grâce à l'ordinateur. Le courrier électronique n'est que l'exemple le plus évident de cette tendance dans la vie des grandes organisations.

S. Zuboff décrit dans son livre (1988) l'effet de cette sorte de communication et de métacommunication constante. Les bases de données contenant et l'enregistrement des transactions et le courrier électronique deviennent le «texte» de l'entreprise. La valeur ajoutée dérive de l'exploitation de ces informations, grâce à la «lecture» de ce «texte», afin de diriger l'activité future de l'entreprise le mieux possible.

Nous assistons sans doute à la constitution d'un nouveau rapport humain avec le langage à l'époque des ordinateurs. L'ordinateur vient en aide à la parole humaine, abolissant la dichotomie classique entre l'écrit (permanent) et le parlé (éphémère), et saisissant celui-ci dans son passage non seulement pour le figer comme écrit mais pour l'analyser le moment venu. Et il s'agit de saisir non seulement la parole, mais cet écrit éphémère, voisin du parlé, qui s'écrivait autrefois à la main sous forme de notes, support de la parole et aide-mémoire. La parole laisse maintenant une trace qui peut se modifier à son tour.

Autrefois, il y avait le domaine de l'écrit, massif, monolithique, immuable. En face, il y avait le domaine de la parole, volatile, fugace, vite oubliée. Les deux domaines ne se rencontraient pas, sauf dans certaines situations institutionnelles (tribunaux, parlements) où la parole avait une valeur surajoutée et donc devait devenir texte; et c'est alors que la technique de la sténographie venait à l'appui, pour saisir le message exact, le verbatim, le mot à mot, dans une transparence voulue parfaite. Et alors le texte de la parole se refermait sur lui-même, immuable, une référence, une permanence, une autorité pour ce qui avait été dit et ne pouvait plus être retiré. (Quod scripsi, scripsi, dit Ponce Pilate aux Juifs.)

Mais si l'écriture venait en aide aux discussions, à la parole, d'une manière inouïe? L'écriture marie désormais la tangibilité de l'écrit-texte avec la volatilité, la mutabilité de la parole. Elle peut intervenir, chez le rapporteur, comme feed-back au cours même de la discussion, et elle donne une portée à la parole qu'elle ne possédait pas auparavant, une dimension nouvelle qui était autrefois le propre des seuls textes: celle de durer, d'être lu par des gens qui n'étaient pas là en temps réel au moment de sa première énonciation. Et cet écrit peut être changé, commenté, élargi par les participants, soit à titre individuel, soit dans des réunions ultérieures.

L'écriture se révèle donc comme le support de la parole, de la discussion : à la fois le moyen d'en amplifier la portée et le moyen de rétroaction sur elle. Mais avec l'ordinateur, il y a même plus. Après le texte informatisé vient I'hypertexte. L'hypertexte est un dispositif permettant des liens entre des éléments de deux ou plusieurs textes différents; pour l'exprimer autrement, l'hypertexte crée non des textes uniques et indépendants, mais des textes sur deux ou plusieurs paliers. Et grâce à I'hypertexte, nous voyons cette transcription, cette activité d'analyse dont nous avons parlé à propos du rapporteur, se proliférer à l'infini, tout au moins virtuelle ment, parce que chaque palier de l'hypertexte peut désormais s'associer, se lier à d'autres paliers. On en parlera plus tard, au moment de l'analyse. 
Après la sténographie, après la bande magnétique transcrite, le travail du rapporteur ouvre la possibilité d'un nouveau rapport de forces entre la parole et l'écrit. Si la parole, au lieu de disparaître une fois énoncée, peut laisser une trace, elle peut se constituer texte. Et si l'écrit, au lieu d'être figé à tout jamais dans l'éternité de l'irrévocable, peut se parer de l'agilité protéenne de la parole, et si cette trace de la parole s'avère capable de subir de multiples transformations, les textes vont devenir infiniment producteurs comme la langue dont parlait W. von Humboldt («aus endlichen Mitteln einen unendlichen Gebrauch machen »).

\section{LE TRAVAIL ANALYTIQUE DU RAPPORTEUR EN TEMPS RÉVERSIBLE}

Lorsque le rapporteur a fait la première partie de son travail, en temps réel, il pourra passer au deuxième stade, celui de l'analyse. Maintenant qu'on a enregistré le texte de la discussion, il s'agit d'en déterminer le sens total. Il faut lui donner une interprétation.

\section{L'étude des textes}

Le rapporteur doit adopter une méthode d'analyse face à ce texte de groupe, cette suite d'énoncés qu'il a notés. Et puisqu'il s'agit bien d'un texte au sens que nous avons précisé, il faudrait regarder du côté de l'analyse des textes pour trouver les éléments d'une méthode. Je citerai quelques essais qui m’ont été directement utiles pour établir ma méthode de rapporteur.

Bursill-Hall (1966) a proposé que les textes dans I'histoire de la linguistique soient étudiés par les méthodes de la linguistique elle-même. La raison en est que les termes techniques utilisés dans les théories d'autrefois ne sont souvent compréhensibles qu'à partir de leur contexte propre. II faut donc étudier les rapports entre les termes, surtout leur coprésence dans les énoncés théoriques, pour définir leur signification. En étudiant les termes techniques employés par les auteurs dans les énoncés où ils apparaissent, et en comparant ces énoncés soigneusement entre eux, on arrive à restituer le système clos, l'arsenal conceptuel des auteurs: «The collocations of these terms represent ordered series of words» (1966: 42). On peut envisager le même type d'analyse dans le cas des termes techniques ou mots clés dans les énoncés du discours rapporté d'une réunion.

Lévi-Strauss (1958), qu'on a cité déjà plusieurs fois, a proposé d'analyser les textes des mythes en les divisant en énoncés comme «unités textuelles». Ensuite, selon lui, il faut procéder à une analyse selon les méthodes de la linguistique structurale. En comparant les énoncés isolés, on peut arriver à des énoncés plus abstraits dont les énoncés recueillis ne seraient que des réalisations ou des variantes. Par exemple, à partir d'énoncés comme «Frdipe épouse sa mère Jocaste» et «Antigone enterre son frère Polynice, violant l'interdiction » on peut déduire la proposition «rapport de parenté surestimé». Ceci est la «grosse unité constitutive» ou «mythème» ou «textème».

Si I'on fait l'analyse des mythes de cette façon, on trouve des «textèmes» coprésents tels que «rapport de parenté surestimé» et «rapport de parenté sousestimé» («Etéocle tue son frère Polynice»; « FEdipe tue son père Laios») (1958: 236). L'on voit par là que les mythes sont typiquement des sortes d'outils logiques faits 
pour surmonter des contradictions de l'expérience vécue. «La pensée mythique procède de la prise de conscience de certaines oppositions et tend à leur médiation progressive» (1958: 248); on peut noter que c'est la règle de la discussion en groupe également. Les «textèmes» extraits par l'analyse du texte ont donc eux-mêmes une cohérence entre eux qui n'est pas celle du texte de départ. «Enfin, si l'on parvient à ordonner une série complète de variantes sous la forme d'un groupe de permutations, on peut espérer découvrir la loi du groupe» (1958: 252). C'est dans ce sens qu'on peut dire que l'analyse du texte donne comme résultante un autre texte, un texte abstrait fait de significations.

Dans le domaine de l'histoire des mentalités, Foucault (1966) en particulier a étudié des corpus de textes dans plusieurs domaines à travers les siècles pour en dégager "l'a priori historique», c'est-à-dire l'ensemble des propositions sous-jacentes qui ont rendu tel ou tel discours savant possible à un moment donné (voir surtout Foucault [1966]). II appelle ce genre d'étude une «archéologie» plutôt qu'une histoire, l'archéologie étant la connaissance du passé grâce à ses monuments, aux objets qui en subsistent. De même, l'étude «archéologique» des textes est une étude des objets qui subsistent en l'absence de leurs auteurs ou créateurs, étude qui cherche à caractériser ces objets d'une manière formelle, les comparer entre eux, et en extraire les tendances et les points communs.

En «archéologue», on étudie les textes venant de plusieurs disciplines qui n'ont peut-être pas de contact évident entre elles, mais qui ont été écrits à une même époque. II ne s'agit pas d'une conversation, d'une discussion dans notre sens, bien sûr, mais d'une série de textes qui traitent le même sujet ou des sujets connexes à un moment donné, d'un discours donc. Le discours d'une époque donnée peut être abordé comme si ces textes disparates étaient l'ouvrage d'un seul auteur. Le discours prend une existence explicite comme une sorte de texte au-dessus des textes. Ou bien, le discours projette, ou reflète, ou enfante un texte qui est le texte de ses lieux communs.

On fait l'analyse de ces textes comme s'il s'agissait d'une discussion, mais une discussion où l'on cherche à dégager non les opinions divergentes (ce qui serait une doxographie) mais l'idéologie sous-jacente, les présupposés partagés et donc non rendus explicites, bref, I'a priori historique. Dans une telle perspective, ce qui importe, ce n'est pas les désaccords, mais l'accord tacite qui rend possible une discussion.

Or, quand un rapporteur a enregistré des discussions, il a devant lui un texte qui peut subir les mêmes stratégies d'analyse qu'un corpus de textes historiques. La discussion a lieu en «temps réel », donc, en temps irréversible. M ais l'enregistrement de la discussion, commel'ensemble des textes, existe bien sûr dans un temps réversible. Ne peut-on pas enrichir la réflexion sur les discussions qui ont eu lieu en temps réel en utilisant des méthodes élaborées à d'autres fins pour dégager l'accord tacite fondamental, l'idéologie, l'univers des valeurs, afin de montrer aux participants où ils sont d'accord même au moment où ils se disputent? De cette façon-là, on pourra dépasser les contradictions en les plaçant dans un contexte plus large d'accord, de consensus, qu'il s'agit justement de rendre explicite. Le «rapport » d'une séance de discussion ne sera donc pas une doxographie, un catalogue des opinions émises, mais une synthèse des valeurs et des notions sous-jacentes qui rendent cette discussion possible.

Je conclus qu'une méthode d'analyse adéquate pour le rapporteur serait une méthode qui permet: 
1. d'identifier les termes techniques de la discussion et de les ranger en séries;

2. de grouper les énoncés dans des «textèmes»; et

3. de dégager les présupposés, les valeurs, les croyances, préalables à toute controverse, à tout désaccord, qui sous-tendent la discussion.

\section{La méthode des notions et propositions}

Voici donc l'exposé sommaire d'une méthode pour analyser le texte des consultations en temps réel.

Ce texte consiste en énoncés rapportés. Un énoncé est une phrase qui a été relevée dans la prise de notes en temps réel. L'énoncé appartient au niveau du temps réel et irréversible.

On procède maintenant à l'identification dans ces énoncés de mots ou de locutions ayant un sens technique, ou qui revêtent une signification surajoutée dans ce contexte spécifique, qui sont définis par le texte, se contrastent avec d'autres, ou qui font partie d'une série de termes. Ce sont des notions. (La notion est déjà le produit de I'analyse; elle peut avoir plusieurs réalisations [synonymes] au niveau de l'énoncé.)

À partir des énoncés également, on construit des propositions. Une proposition est une classe d'énoncés. Les propositions regroupent les notions, et en définissent les rapports. Elles contribuent en même temps à la définition des notions.

Les propositions sont analysées en classes de propositions ou textèmes. (Contrairement à Lévi-Strauss, je formule ceux-ci comme des phrases entières ayant la même structure syntaxique que les énoncés de départ.) Voilà des propositions qui résument le contenu de toute une série de propositions.

De même, les notions sont analysées en classes de notions. Ces classes de notions résument des séries de notions qui sont liées sémantiquement.

Les propositions, classes de propositions, notions et classes de notions appartiennent au niveau du temps réversible, donc, à celui de l'analyse.

Ducrot, dans son Dire et ne pas dire, écrit: «la communication linguistique n'est pas que la transmission d'informations; le discours est incompréhensible pour une sémantique qui se tiendrait au niveau de l'explicite». II y a une autre classe de propositions, qui n'apparaît pas dans le texte de la discussion. Ce sont les propositions tacites ou implicites. Chacune de ces propositions tacites dérive par implication logique d'une proposition explicite. De telles formulations aident notamment à exprimer les perceptions, les croyances et les valeurs sous-jacentes des participants, bref, leur pensée intime. II est capital d'exprimer cette pensée intime, parce que souvent on trouve avec des groupes en conflit qu'ils habitent dans des univers de pensée différents. II faut rendre explicites leurs présupposés, pour les comprendre, les faire comprendre, et enfin pour résoudre le conflit.

Propositions contenant des notions, classes de propositions contenant des classes denotions, enfin propositions implicites: nous sommes maintenant en présence d'un texte à la deuxième puissance, d'un métatexte si l'on veut, qui précise l'ensemble des valeurs, des croyances, des a priori, des présupposés, des perceptions du monde, des savoirs sur le sujet en question au moment de la discussion. 


\section{Commentaire, niveau de texte}

Ce n'est pas tout. II y a un niveau supplémentaire d'analyse. Le rapporteur peut fournir un commentaire de la discussion et de l'interprétation. Cet espace de commentaire peut se créer très tôt, mais ne pas forcément être partagé avec les participants. II se fera typiquement «dans les coulisses». Les commentaires comprennent des gloses sur les notions, sur les propositions, ou sur les rapports hiérarchiques qui existent entre celles-ci.

\section{Hypertexte}

Cette structuration en niveaux d'analyse est rendue possible par l'hypertexte.

L'hypertexte, qu'Internet a fait connaître, est un texte à plusieurs niveaux, avec des liens entre des textes entiers, ou des mots ou phrases. (On consultera avec profit Bolter [1991] sur les possibilités de l'écriture hypertextuelle.) Le problème en ce qui concerne les niveaux d'analyse est qu'ils tendent à s'effacer les uns les autres. C'est-àdire que le nouveau niveau d'abstraction et d'analyse tend à effacer ou à remplacer le précédent. On constate la même chose avec la traduction: la traduction remplace I'original dans l'esprit du lecteur qui la lit. Le travail du traducteur aboutit à un texte «d'arrivée» qui remplace le texte «de départ». Comme l'a dit Folkart: «Qui dit travail dit irréversibilité» (1991 : 359). M ais il y a maintenant la possibilité de niveaux de texte qui ne se remplacent pas ni ne s'effacent, mais qui se complètent et qui s'appellent les uns les autres: voilà I'hypertexte.

L'hypertexte permet une certaine réversibilité. La parole, nous l'avons vu, est le domaine de l'irréversible. Le travail de la réénonciation est elle aussi irréversible. Toutefois, I'hypertexte conserve les niveaux antérieurs de l'analyse. Un niveau n'efface pas le niveau qui l'a précédé. Le lecteur du travail du rapporteur peut reconstituer toutes les étapes de l'analyse s'il le veut. II peut commencer avec le métatexte comprenant les classes de notions et de propositions, et retracer le chemin de l'analyse jusqu'à retrouver les énoncés de l'enregistrement initial.

\section{Le rapport final}

La résultante de toutel'analyse est le rapport final. Conclusion apparemment banale: à la fin de tout travail de consultant il y a toujours un rapport final, et il ne s'agit généralement que d'un compte rendu de ce qui s'est passé, et d'une liste de conclusions ou de recommandations. Mais le rapporteur, à mon sens, doit produire une sorte différente de rapport: un rapport qui sera hypertextuel, unissant les niveaux de I'analyse. De ce fait, un lecteur averti pourra étudier telle notion ou telle proposition en traçant ses réalisations à chaque moment de l'analyse. II pourra même ajouter ses propres commentaires, voire les résultats de discussions ultérieures. Le rapport final devient un texte vraiment «vivant», «ouvert», qui en principe n'est jamais achevé, qui ne se referme pas comme un livre, immuable, sourd à toute question, à tout dialogue ultérieur... 


\section{LE RAPPORTEUR : UNE PROFESSION LINGUISTIQUE}

La méthode que je viens de décrire a fait ses preuves dans la consultation interne². Elle serait à envisager pour les consultations externes de grande envergure, notamment les commissions gouvernementales où l'on sollicite les opinions d'un grand nombre de citoyens.

Pour ce faire, il faudrait envisager des équipes de rapporteurs assurant l'enregistrement et I'analyse des discussions. Ce qui nous amène à considérer, en terminant, les aptitudes et la formation qu'il faut pour pouvoir désigner des rapporteurs pour ce genre de travail.

Je crois qu'on peut former des rapporteurs comme on forme les interprètes de conférence. Bien entendu, les personnes qu'on choisit pour cette formation devraient avoir certaines compétences de départ. En tout cas, à la fin d'une période de formation, elles devront:

- avoir une très bonne connaissance de la langue de travail, tant orale qu'écrite;

- avoir une grande concentration, la discipline de l'écoute;

- avoir une excellente culture générale, pouvoir s'informer rapidement sur le sujet qui sera traité dans la réunion, et surtout se doter du vocabulaire technique dont se serviront les participants;

- savoir prendre des notes très détaillées;

- savoir manier l'ordinateur, et avoir une compréhension des possibilités de l'hypertexte;

- être initiées aux techniques linguistiques pour le stade de l'analyse.

En somme, le rapporteur doit avoir un «esprit de finesse» pascalien pour saisir les sens et les rapports dans les interactions, surtout des sens implicites. M ais il doit en même temps avoir un «esprit de géométrie», pour pouvoir arranger toutes ces pensées qu'il entend en un exposé logique.

La formation des rapporteurs sera fondée sans doute sur celle des interprètes de conférence décrite par Seleskovitch et Lederer (1986). Les futurs rapporteurs devront étendre leur culture générale, «apprendre à apprendre». II leur faudra des exercices pour écouter longtemps, pour rendre le sens par la suite; des exercices pour apprendre à «lire» une interaction (ce seront des exercices avec vidéo, sans doute). Les futurs rapporteurs feront des études de terminologie, souvent des études sur le terrain, où il faudra agir comme un ethnologue: interviewer des «informateurs indigènes». II faudra connaître l'éthique, l'idéologie de divers groupes professionnels ou nationaux, et savoir gagner la confiance de ces «indigènes».

Dans tout l'exposé qui précède, j'ai utilisé la discussion savante du rôle de l'interprète de conférence pour penser, élaborer et systématiser le rôle du rapporteur de conférence. Ce faisant, j'espère contribuer à la compréhension de tous ces rôles: non seulement celui du rapporteur, mais ceux de l'interprète de conférence, du traducteur, du terminologue. C'est que je vois le rapporteur comme une profession linguistique parmi ces autres. 


\section{NOTES}

1. Pour le Canada, il faudrait consulter Citizens' Forum on Canada's Future: Report to the People and Government of Canada, Ottawa, Supply and Services, 1991; Porteous, W., Citizens' Forum on Canada's Future: Report on the Consultation Process, Ottawa, Canadian Centre for Management Development/Centre canadien de la gestion, 1992.

2. La technique du rapport exposée dans cet article a été élaborée lors d'une consultation interne de la fonction publique menée par le Vérificateur Général (ministère des Finances) de la ColombieBritannique en 1987. 350 cadres ont participé à 15 réunions à travers la province.

\section{RÉFÉRENCES}

Bolt er , J. D. (1991) : Writing Spaces, Hillsdale (NJ), L. Erlbaum Associates.

Bur sil I-H al I, G. L. (1966) : «Notes on the Semantics of Linguistic Description », dans Bazel I et Al. (Eds.), In M emory of J. R. Firth, London, Longmans.

Corr adi Fiumara, G. (1990) : La filosofia dell' ascolto, Milan.

Ducr ot , O. (1980) : Dire et ne pas dire: principes de sémantique linguistique, Paris, Hermann.

Fol kart, B. (1991) : Le conflit des énonciations: traduction et discours rapporté, Québec, Balzac.

Fo u cault, M. (1966) : Les mots et les choses: une archéologie des sciences humaines, Paris, Gallimard.

Leder er , M . (1981) : La traduction simultanée: expérience et théorie, Paris, M inard.

Lév i-St r auss, C. (1958) : "La structure des mythes», dans Anthropologie Structurale, Paris, Plon.

M acN a mee, T. (1984) : «La terminologie de la neurolinguistique: perspectives diachroniques», M eta, 29 (1), p. 91-98.

M ossop, B. (1984) : «The Translator as Rapporteur: a Concept for Training and Selfimprovement», M eta, 28 (3), p. 244-278.

M o sso p, B. (1987) : «Who IsAddressing Us When We Read a Translation ?», TextConText, 1, p. 1-22.

Sel esk ov it ch, D. (1968) : L'interprète dans les conférences internationales: problèmes de langage et de communication, Paris, $M$ inard.

Sel eskovit ch, D. et M. Leder er (1986) : Interpréter pour traduire, $2^{e}$ édition, Paris, Didier Érudition.

Zuboff, S. (1988) : In the Age of the Smart Machine: the Future of Work and Power, New York, Basic Books. 